\title{
Acute Effect of Basketball-Specific Exercise on Lower Limb Injury Risk Mechanisms in Male Basketball Players U16 and U18
}

\author{
Michal Lehnert ${ }^{1 *}$, Karel Hůlka ${ }^{1}$, Mark De Ste Croix ${ }^{2}$ and Kristýna Horutová ${ }^{1}$ \\ ${ }^{1}$ Faculty of Physical Culture, Palacký University in Olomouc, Czech Republic \\ ${ }^{2}$ Exercise and Sport Research Centre, University of Gloucestershire, United Kingdom
}

*Corresponding author: Michal Lehnert, PhD, Faculty of Physical Culture, Palacký University, Tr Miru 117, 771-11 Olomouc, Czech Republic

Submission: 非 March 19, 2018; Published: 梅 April 05, 2018

\begin{abstract}
The aim of this study was to examine and compare the acute effects of basketball-specific fatigue on muscular stiffness and reactive strength in male adolescent basketball players of two age categories. Reactive strength, expressed by means of the reactive strength index (RSI), and leg stiffness (LS) as mechanisms associated with the risk of ACL injury were measured in eleven players of the U16 category (age $16.1 \pm 0.4$ years; body height $185 \pm 6.5 \mathrm{~cm}$; body mass $74.3 \pm 9.9 \mathrm{~kg}$ ) and ten players of the U18 category (age $17.7 \pm 0.4$ years; body height $187 \pm 5.7 \mathrm{~cm}$; body mass $79.7 \pm 7.4 \mathrm{~kg}$ ) pre and post simulated basketball match play $\left(\mathrm{SBFP}_{28}\right)$. RSI was determined by a drop jump test, LS by a 20 sub-maximal two-legged hopping test. No significant effects of SBFP28 on the monitored parameters were observed with the exception of RSI in U16 ( $p=0.013, r=0.53$ ). Similarly, there were no significant differences in the fatigue-related responses to $\mathrm{SBFP}_{28}$ for RSI and LS between the age groups. These results indicated that irrespective of age, the stabilization function of the knee muscles was not impaired and consequently the risk of ACL injury was not increased.
\end{abstract}

Keywords: Youth; Fatigue; Neuromuscular; Reactive strength; Stiffness

\section{Introduction}

Basketball is a physically demanding sport, which imposes a highly variable load on the players [1]. Basketball performance combines maximum intensity activities and medium or low intensity multidirectional movements such as acceleration and deceleration, pivoting, dribbling, shuffling, jumping and landing, often performed unilaterally [2,3]. For these reasons, youth basketball belongs among risky sports with frequent lower limb injuries including injuries of the knee joint and hamstrings [4]. In 70 to 80 percent of cases, the injury is of a non-contact nature [5]. In particular, anterior cruciate ligament (ACL) injuries are associated with the longest interruption of training and competition, and also predispose to recurrence and other difficulties including earlier degenerative changes $[5,6]$. Non-contact ACL injuries appear to predominantly occur in a rapid change of direction initiated on one leg, landing from a jump on one leg, and pivoting [6-8]. It was proved that injury risk increases with chronological age and may also be related to important periods during growth and maturation [9]. It was recognized in youth athletes that 16 to 18-year olds represent a group at greatest risk where most injuries are likely to occur [10], which was also confirmed in sports such as basketball and soccer [11]. Other recent studies of youth reported the highest incidence of injury in the group of 13 to 15-year olds, followed by 16 to18 year olds [12], and the highest injury incidence around the time of Peak Height Velocity [13].

It was also documented that muscle fatigue is one of the modifiable risk factors of ACL injury [14,15]. Injury incidence data show an increased risk of injury towards the end of matches, when fatigue is most likely to be present [16]. There are a number of mechanisms that relate to an increased risk of knee injury and it was documented that muscular and neuromuscular control is important in reducing load on ACL and thus reducing the risk of ACL injury $[17,18]$. Fatigue decreases the neuromuscular component of the knee dynamic stability and thus increases the risk of ACL injury $[19,20]$. In fatigue-related prevention of ACL injuries, a significant indicator is muscle stiffness [21]. Information about lower limb stiffness is particularly important in terms of risk of injury resulting from a jump or change of direction impact [17]. Currently, there are only few studies focusing on the effect of fatigue on lower limb stiffness, mostly in soccer. Some studies claim that lower limb stiffness is compromised as a result of fatigue [22,23], while others 
report individualized changes in lower limb stiffness after a fatigue protocol [24]. It was also suggested that the influence of age on the ability to perform eccentric-concentric movements exists and that adults are capable of greater pre-activation and forward control compared with children and adolescents [25-27]. This suggests that children and adolescents represent a risk group in terms of injury [17].

Fatigue may also lead to changes in reactive strength of leg muscles [28]. The level of reactive strength in individuals is assessed by means of the reactive strength index (RSI). RSI represents an individual ability of transition from eccentric to concentric muscle contraction [29] and is determined for monitoring stress exerted on the muscle-tendon complex during plyometric exercise. RSI is also a significant indicator in the prevention of ACL injuries [30]. Studies dealing with the effect of age on the results of RSI point to gradually improving results during adolescence [26,27]. However, there are only few studies dealing with RSI as a result of fatigue in youth athletes, and on top of that with conflicting findings $[23,31]$. The above-mentioned findings on the occurrence of ACL injuries in team sports indicate that this is a serious phenomenon in which an important role plays the etiology of muscular and neuromuscular control, fatigue, and age. Nevertheless, there is a paucity of research on the influence of acute fatigue from match-play on physiological mechanisms in adolescent populations. Therefore, this study aims to examine and compare the acute effects of basketballspecific fatigue on muscular stiffness and reactive strength in male adolescent basketball players of two age categories.

\section{Methods}

\section{Participants}

The study involved a group of youth basketball players of the U16 category $(\mathrm{N}=11$; age $16.1 \pm 0.4$ years; body height $185 \pm 6.5$; body mass $74.3 \pm 9.9 \mathrm{~kg}$ ) and $\mathrm{U} 18$ category $(\mathrm{N}=10$; age $17.7 \pm 0.4$ years; body height $187 \pm 5.7 \mathrm{~cm}$; body mass $79.7 \pm 7.4 \mathrm{~kg}$ ). The players trained on average six times per week and played one competitive match per week during the competitive season. All players tested were fully informed about the aim of the study and the testing procedures. The day before testing, the players were not exposed to any training load. The leg preferred for kicking was considered the dominant leg (DL) and the contra lateral leg as the non-dominant leg (NL). The study was approved by the institution's ethics committee and conformed to the Declaration of Helsinki regarding the use of human subjects. All players tested were free of any musculoskeletal lower-extremity injuries and were fully informed about the aim of the study and the testing procedures. A written informed consent to the testing procedures and the use of the data for further research was obtained from the players' parents.

\section{Procedures}

The measurements took place during the 2015 pre-season. During the first session, a week before testing, the players underwent anthropometric measurements, familiarization with the fatigue protocol, and the tests applied. During the second session, before the testing procedure, the players completed non-specific warm-up exercises, which included cycling on a stationary bicycle ergometer for 5 minutes at $1.5 \mathrm{~W} / \mathrm{kg}$, dynamic stretching exercises for six minutes, which targeted the main muscle groups involved during the test, and finally fifteen squats. The warm-up routine was performed under the researcher's supervision. The subsequent testing procedure consisted of measuring reactive strength, leg stiffness, and isokinetic strength. Then the players performed the fatigue protocol. Immediately after the fatigue protocol, the tests were repeated in the same order.

\section{Reactive strength}

The reactive strength index (RSI) was determined by means of a drop jump test with hands on the hips. The vertical jump performance was assessed using a portable optical timing system Opto jump Next (Microgate, Bolzano, Italy) with manufacturerdeclared accuracy of $0.001 \mathrm{~s}$. The players jumped from a $30 \mathrm{~cm}$ high box in order to perform a maximum jump for the shortest period of take-off [32]. To familiarize the players with the testing procedure, two trials were performed followed by three measured attempts with a 30s rest interval between the sets. The greatest value of the three attempts was used in further analyses. RSI was calculated as the ratio between jump height and contact time on the floor [33].

\section{Leg stiffness}

To determine stiffness of the lower limbs, all players performed one trial set and two measured sets of 20 sub-maximal two-legged hopping using the Opto jump Next system (Microgate, Bolzano, Italy) with hands on the hips and the torso in an upright position. The frequency of hopping was maintained at $2.5 \mathrm{~Hz}$ by using a mechanical metronome (Wittner GmbH \& Co. KG, Isny, Germany). Of the two sets performed, the set in which the frequency of jumps was closest to the frequency determined by the metronome was selected. From this set, the ten consecutive jumps that were closest to the determined frequency of hopping were used for further analyses [34]. There was a 1-minute rest interval between the sets. Both absolute leg stiffness (ABSLS; $\mathrm{kN} \cdot \mathrm{m}-1$ ) and relative leg stiffness (RELLS; $\mathrm{kN} \cdot \mathrm{m}-1$ ), i.e. normalization of absolute leg stiffness according to leg length and body mass, were calculated [32].

\section{Specific basketball fatigue protocol $\left(\mathrm{SBFP}_{28}\right)$}

The data are collected over four separate periods as in a match. There is a two-minute break following the first and third periods and a fifteen-minute break following the second period [35]. Each period consists of seventeen activity sections involving maximal activity (sprints), submaximal activity (running at $35 \mathrm{~m} . \mathrm{s}^{-}$ ${ }^{1}$ ), low 3-5 intensity activity (jogging and walking at $1.5-3 \mathrm{~m} . \mathrm{s}^{-1}$ ) and standing (for 15s). The first maximum intensity section starts when a whistle is blown and the time is measured by electronic timing gates (PR1aW, Alge-Timing GmbH, Austria) with one-hundredth of a second accuracy. The scheme of the test is depicted in Figure 1. 


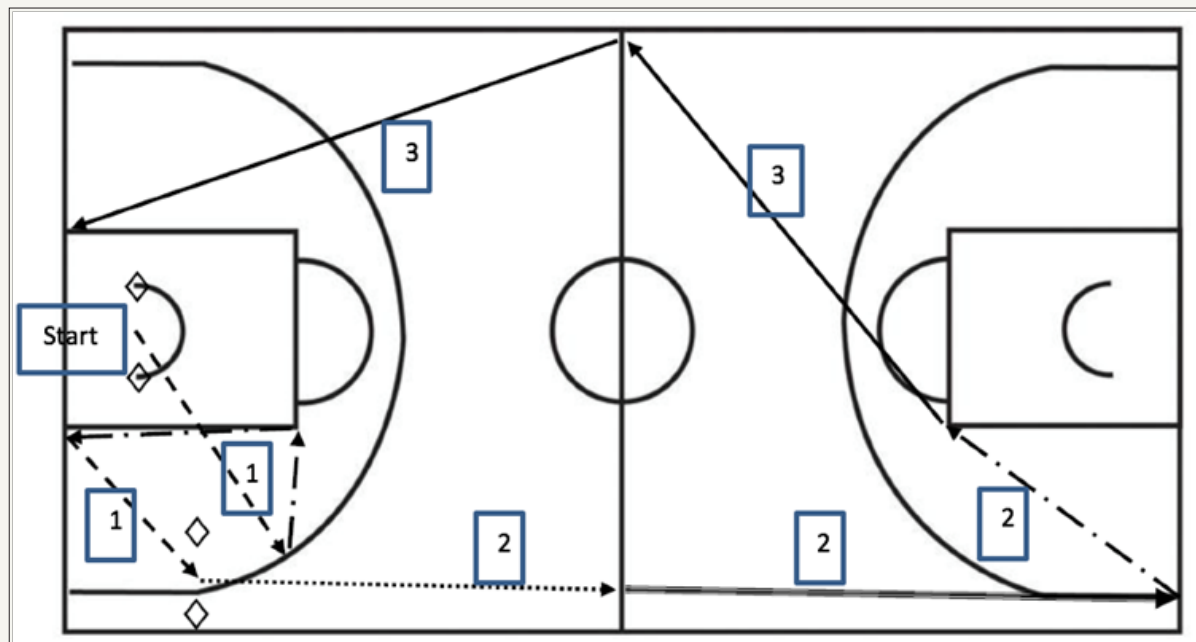

Figure 1: Measured sections of $\mathrm{SBFP}_{28}$.

$\diamond:$ Photocells; $\Longrightarrow$ : Backpedalling;--- $\rightarrow$ : Sprinting; Activity; 2: Medium Intensity Activity; 3: Low Intensity Activity

\section{Statistical analysis}

The distributions of the data sets were checked for homogeneity and skewness using the Kolgomorov-Smirnov test. Descriptive statistics (means, medians and standard deviations) were calculated for all variables. Wilcoxon nonparametric tests were used to examine whether the basketball specific fatigue protocol led to significant changes in the variables observed. Mann-
Whitney tests were used to compare the differences between the results of the U16 and U18 groups. The effect size was determined by the Cohen's $d$ [36] and classified as small $(0.00 \leq d \leq 0.49)$, medium $(0.50 \leq d \leq 0.79)$, and large $(d \geq 0.80)$. The significance level was set at $\mathrm{P}<0.05$. A statistical analysis was performed using the data analysis software system Statistica, version 12 (Stat Soft, Inc., Tulsa, USA).

\section{Results}

Table 1: Monitored parameters of pre and post basketball specific fatigue protocol $\mathrm{SBFP}_{28}$ in the group of U16 basketball players.

\begin{tabular}{|c|c|c|c|c|c|c|}
\hline Parameter & $\mathbf{N}$ & Pre Test M $\mathbf{S D}$ & Post Test M \pm SD & \% Change & Z & p \\
\hline ABSLS & 11 & $26.65 \pm 5.04$ & $25.84 \pm 4.48$ & 96.9 & 0.533 & 0.594 \\
\hline RELLS & 11 & $32.63 \pm 7.28$ & $31.35 \pm 4.97$ & 96.1 & 0.622 & 0.534 \\
\hline RSI & 11 & $0.49 \pm 0.08$ & $0.54 \pm 0.13$ & 110.7 & 2.49 & $0.013^{*}$ \\
\hline
\end{tabular}

Table Abbreviations: N: Number of Players; M: Mean; SD: Standard Deviation; ABSLS: Absolute Leg Stiffness; RELLS: Relative Leg Stiffness; RSI: Reactive Strength Index; DL: Dominant Leg; NL: Non-Dominant Leg; H/Q: Hamstring Eccentric-to-Quadriceps Concentric Functional Ratio; 60: Angular Velocity of $60^{\circ} / \mathrm{s} ; 180$ : Angular Velocity of $180^{\circ} / \mathrm{s}$; Z: Wilcoxon Test Criterion; *: Significant Difference Between Pre and Post Test $(\mathrm{p}<0.05)$

Table 2: Monitored parameters of pre and post basketball specific fatigue protocol $\mathrm{SBFP}_{28}$ in the group of U18 basketball players.

\begin{tabular}{|c|c|c|c|c|c|c|}
\hline Parameter & $\mathbf{N}$ & Pre Test M \pm SD & Post Test M \pm SD & \% change & Z & p \\
\hline ABSLS & 10 & $30.96 \pm 6.68$ & $29.56 \pm 6.18$ & 95.5 & 1.172 & 0.241 \\
\hline RELLS & 10 & $35.29 \pm 6.42$ & $33.79 \pm 6.58$ & 95.8 & 1.07 & 0.284 \\
\hline RSI & 10 & $0.41 \pm 0.07$ & $0.41 \pm 0.07$ & 100 & 0.05 & 0.959 \\
\hline
\end{tabular}

Table Abbreviations: N: Number of Players; M: Mean; SD: Standard Deviation; ABSLS: Absolute Leg Stiffness; RELLS: Relative Leg Stiffness; RSI: Reactive Strength Index; DL: Dominant Leg; NL: Non-Dominant Leg; H/ Q: Hamstring Eccentric-to-Quadriceps Concentric Functional Ratio; 60: Angular Velocity of $60^{\circ} / \mathrm{s}$; 180: Angular Velocity of $180^{\circ} / \mathrm{s}$; Z: Wilcoxon Test Criterion

The values of measured variables of the pre- and post- $\mathrm{SBFP}_{28}$ and their changes for U16 and U18 groups of basketball players are reported in Table $1 \& 2$. No significant effects of $\mathrm{SBFP}_{28}$ on the monitored parameters were evident with the exception of RSI for $\mathrm{U} 16(\mathrm{p}=0.013, \mathrm{r}=0.53)$. The between-group differences in the changes of the monitored parameters are reported in Table 3 . There were no significant differences in the fatigue-related responses to $\mathrm{SBFP}_{28}$ for any parameters between the groups. 
Table 3: Differences in changes of monitored parameters of pre and post basketball specific fatigue protocol $\mathrm{SBFP}_{28}$ between the U16 and $\mathrm{U} 18$ groups.

\begin{tabular}{|c|c|c|c|c|c|c|c|}
\hline Parameter & U16 M & Mdn & SD & U18 M & Mdn & SD & p \\
\hline$\Delta$ ABSLS 1-2 & 0.81 & 0.73 & 3.92 & 1.46 & 0.7 & 4.65 & 0.972 \\
\hline$\Delta$ RELLS 1-2 & 1.29 & 0.71 & 5.03 & 1.55 & 0.74 & 5.4 & 0.86 \\
\hline$\Delta$ RSI 1-2 & -0.05 & -0.03 & 0.06 & -0.03 & -0.03 & 0.03 & 0.503 \\
\hline
\end{tabular}

Table Abbreviations: N: Number of Players; M: Mean; SD: Standard Deviation; ABSLS: Absolute Leg Stiffness; RELLS: Relative Leg Stiffness; RSI: Reactive Strength Index; DL: Dominant Leg; NL: Non-Dominant Leg; H/Q: Hamstring Eccentric-to-Quadriceps Concentric Functional Ratio; 60: Angular Velocity of $60^{\circ} / \mathrm{s} ; 180$ : Angular Velocity of $180^{\circ} / \mathrm{s} ; \Delta$ : Absolute Change

\section{Discussion}

The main finding of the current study is that there were no significant age differences in the fatigue-related responses to $\mathrm{SBFP}_{28}$ for any of the parameters. Another finding is that $\mathrm{SBFP}_{28}$, which was designed as a basketball-specific match simulation protocol, did not significantly compromise any of the observed parameters in any of the groups of youth basketball players. Oppositely, RSI values significantly increased in the U16 group. These results point out that after the basketball-specific fatigue protocol, the players' ability to effectively utilize neuromuscular mechanisms to control joint movement and reduce load on the ligaments was not compromised, which suggests that injury risk did not increase in the players of both groups due to simulated match-play. The results of this study did not show fatigue-related changes in ABSLS or RELLS in the U16 and U18 categories. These results indicate that the fatigue protocol did not compromise neuromuscular pre-activation and neuromuscular feed-forward control (anticipation of known stimuli characterized by preventive muscle activity) as an indicator of dynamic stability of the knee. Thus it can be assumed that the ability of the muscles to resist movement within the tibiofemoral joint (tibiofemoral shear movements), which prevents ACL from being under strain [21] did not decrease.

Studies examining the effects of simulated match-play on muscular and neuromuscular capability in youth basketball players are missing in the literature. Nevertheless, there are a few studies examining the effects of simulated match-play on muscular and neuromuscular capability in male youth soccer players and the results of these studies are ambiguous. In some cases leg stiffness was compromised [23,31]; some authors reported a nonsignificant decrease in ABSLS [24]. In the latter study, the authors also identified that altered stiffness due to simulated match play is individualised (in half of the pro-bands the value increased, in the other half the value decreased).

A comparison of the results of the above mentioned studies suggests that the differences may be influenced by the specifics of fatigue protocols for individual sports (but also within a particular sport), different levels of training of the monitored groups, gender differences, individual responses to the fatigue exercise, etc. Simillarly to youth, the results of studies in adult athletes did not bring coherent results. While leg stiffness decreased after sportspecific fatigue protocol in soccer [22], highly individual changes were reported in runners [37]. Also in the case of reactive strength evaluated by RSI, the results did not show a negative effect of $\mathrm{SBFP}_{28}$. In the U16 group, the authors of the present study even recorded a significant improvement in RSI $(p=0.013)$. The significance of the changes after completing SBFP28 is also confirmed by the "effect size" value ( $\mathrm{r}=0.53$; large effect). The results suggest that the ability to change eccentric muscular action to concentric in a stretch-shortening cycle was not compromised in the players. This indicates that the players demonstrated a good ability of the muscle-tendon unit to cope with the stress induced by $\mathrm{SBFP}_{28}$. Considering that jumping and landing are important aspects of basketball-specific fitness and that RSI was determined by means of a drop jump test, it could be expected, that these findings are also positive from the game performance perspective, specifically in terms of the quality of repeated jumping and lending actions. The findings of the current study correspond to the results of a recent study on elite adolescent soccer players (age $15.7 \pm 0.5$ years), in which RSI increased significantly following a simulated soccer match play [31]. However, in a similar study on elite youth soccer players (age $14.4 \pm 0.5$ years), RSI decreased significantly.

In the current study, the authors did not observe significant age differences in the fatigue-related responses to $\mathrm{SBFP}_{28}$, either in leg stiffness (ABSLS; RELLS) or RSI. As mentioned above, these results cannot be compared with studies on youth basketball players. Nevertheless, in a later study on youth soccer players aged 13 to 16 years, the authors did not report any significant differences in fatigue-related responses to competitive match play for the parameters observed including ABSLS, RELLS, and RSI between the two age subgroups; $13.6 \pm 0.3$ years and $15.8 \pm 0.1$ years. Oppositely, a recent study on female youth soccer players indicated that leg stiffness decreased as a result of post simulated soccer in U13, but did not change in the U15, and increased in the U17 [38]. The data obtained in the present study also indicate that the values of both leg stiffness and RSI were higher in the U18 category compared with U16, in both pre-and post-test. This supports the suggestion that the ability to perform eccentric-concentric movements is positively influenced by age and that the older category has better dynamic stability of the knee joint and consequently a lower risk of ACL injury in sports activities [26,27]. The observed tendency also corresponds to the findings of a later study [25], in which the authors found out a significant increase in leg stiffness between 11 and 20 years of age in boys, with the highest increments between 17 and 20 years. One of the limitations of the study is that the indicators of neuromuscular fatigue were measured only after the 
fatigue protocol. Another limitation is a low number of players who were involved in the study.

\section{Conclusion}

The $\mathrm{SBFP}_{28}$ did not induce a significant reduction either in leg stiffness or reactive strength in the observed group of male adolescent basketball players. The results indicated that irrespective of age, the stabilization function of the knee muscles was not impaired and consequently the risk of ACL injury was not increased. However, a small number of players, and a relatively small age difference and other aspects of the methodological design of the study need to be considered. Due to the lack of similar studies on young basketball players and the results of a small number of previously conducted studies on youth in other sports, further research into the impact of the acute effect of match simulation protocol or competitive match-play on the mechanisms associated with the risk of ACL injury in youth basketball players is needed.

\section{Acknowledgement}

This study was supported by research grant from the Czech Science Foundation NO. 16-13750S.

\section{References}

1. Hůlka K, Cuberek R, Bělka J (2013) Heartrate and time-motion analyses in top junior players during basketball matches. Acta Univ Palacki Olomuc, Gymn 43(3): 27-35.

2. Apostolidis N, Nassis GP, Bolatoglou T, Geladas ND (2004) Physiological and technical characteristics of elite young basketball players. J Sports Med Phys Fitness 44(2): 157-163.

3. Narazaki K, Berg K, Stergiou N, Chen B (2009) Physiological demands of competitive basketball. Scand J Med Sci Sports 19(3): 425-432.

4. Schwebel DC, Brezausek CM (2014) Child development and pediatric sport and recreational injuries by age. J Athl Train 49(6): 780-785.

5. Alentorn-Geli E, Myer GD, Silvers HJ, Samitier G, Romero DR, et al. (2009) Prevention of non-contact anterior cruciate ligament injuries in soccer players. Part 1: Mechanisms of injury and underlying risk factors. Knee Surg Sports Traumatol Arthrosc 17(7): 705-729.

6. Thacker SB, Stroup DF, Branche CM, Gilchrist J, Goodman RA, et al. (2003) Prevention of knee injuries in sports: a systematic review of the literature. J Sports Med Phys Fitness 43(2): 165-179.

7. Cochrane JL, Lloyd DG, Buttfield A, Seward H, McGivern J (2007) Characteristics of anterior cruciate ligament injuries in Australian football. J Sci Med Sport 10(2): 96-104.

8. Silvers HJ, Mandelbaum BR (2007) Prevention of anterior cruciate ligament injury in the female athlete. Br J Sports Med 41(1): 152-159.

9. Parkkari J, Pasanen K, Mattila VM, Kannus P, Rimpelä A (2008) The risk for a cruciate ligament injury of the knee in adolescents and young adults: a population-based cohort study of 46500 people with a 9 year follow-up. Br J Sports Med 42(6): 422-426.

10. Shea KG, Pfeiffer R, Wang JH, Curtin M, Apel PJ (2004) Anterior cruciate ligament injury in pediatric and adolescent soccer players: An analysis of insurance data. J Pediatr Orthop 24(6): 623-628.

11. Lohmander LS, Englund PM, Dahl LL, Roos EM (2007) The long-term consequence of anterior cruciate ligament and meniscus injuries: osteoarthritis. AM J Sports Med 35(10): 1756-1769.
12. Rumpf MC, Cronin J (2012) Injury incidence, body site, and severity in soccer players aged 6-18 years: Implications for injury prevention. Stren Cond J 34(1): 20-31.

13. Van der Sluis A, Elferink-Gemser M, Coelho-e-Silva MJ, Nijboer JA, Brink MS, et al. (2014) Sport injuries aligned to peak height velocity in talented pubertal soccer players. Int J Sports Med 35(4): 351-355.

14. Dai B, Mao D, Garrett WE, Yu B (2014) Anterior cruciateligament injuries in soccer: Loading mechanisms, risk factors, and prevention programs. J Sport Health Sci 3(4): 299-306.

15. Sangnier S, Tourny-Chollet C (2007) Comparison of the decrease in strength between hamstrings and quadriceps during isokinetic fatigue testing in semiprofessional soccer players. Int J Sports Med 28(11): 952957.

16. Ekstränd J, Hägglund M, Waldén M (2009) Injury incidence and injury patterns in professional football: The UEFA injury study. Br J Sports Med 45(7): 553-558

17. Oliver J, Smith P (2010) Neuralcontrolof leg stiffness during hopping in boys and men. J Electromyogr Kinesiol 20(5): 973-979.

18. Hawkins RD, Hulse MA, Wilkinson C, Hodson A, Gibson M (2001) The association football medical research programme: an audit of injuries in professional football. Br J Sports Med 35(1): 43-47.

19. Rozzi SL, Lephart SM, Fu FH (1999) Effects of muscular fatigue on knee joint laxity and neuromuscular characteristics of male and female athletes. J Athl Train 34(2): 106-114

20. Smith HC, Vacek P, Johnson RJ, Slauterbeck JR, Hashemi J, et al. (2012) Risk factors for anteriorcruciateligament injury: A review of the literature-Part 1: Neuromuscular and anatomic risk. Sports Health 4(1): 69-78.

21. Hughes G, Watkins J (2006) A risk-factor model for anterior cruciate ligament injury. Sports Med 36(5): 411-428.

22. Dutto DJ, Smith GA (2002) Changes in spring-mass characteristics during treadmill running to exhaustion. Med Sci Sports Exerc 34(8): 1324-1331.

23. Lehnert M, De SteCroix M, Zaatar A (2017) Muscular and neuromuscular control following soccer-specific exercise in youth male footballers: Changes in injury risk mechanisms. Scand J Med SciSports 27(9): 975982.

24. Oliver JL, De SteCroix, MBA, Lloyd RS (2014) Altered neuromuscular control of leg stiffness following soccer-specific exercise. Eur J AppPhysiol 114(11): 2241-2249.

25. Laffaye G, Choukou MA, Benguigui N, Padulo J (2017) Age and genderrelated development of stretch shortening cycle during a sub-maximal hopping task. Biol Sport 34(1): 29-35.

26. Lloyd RS, Oliver JL, Hughes MG, Williams CA (2011) The influence of chronological age on periods of accelerated adaptation of stretchshortening cycle performance in pre and postpubescent boys. J Stren Cond Res 25(7): 1889-1897.

27. Lloyd R, Oliver J, Hughes M, Williams C (2012) Age-related differences in the neural regulation of stretch-shortening cycle activities in male youths during maximal and sub-maximal hopping. J Electromyogr Kinesiol 22(1): 37-43

28. Toumi H, Poumarat G, Best TM, Martin A, Fairclough J, et al. (2006) Fatigue and muscle-tendon stiffness after stretch-shortening cycle and isometric exercise. Appl Physiol Nutr Metab 31(5): 565-572.

29. Young W (1995) Laboratory strength assessment of athletes. New Stud Athl 10(1): 88-96. 
30. Raschner C, Platzer HP, Patterson C, Werner I, Huber R, et al. (2012) The relationship between ACL injuries and physical fitness in young competitive ski racers: a 10-year longitudinal study. Br J Sports Med 46(15): 1065-1071.

31. Lehnert M, De SteCroix M, Xaverova Z, Botek M, Varekova R, et al. (2018) Changes in injury risk mechanisms after soccer specific fatigue in male youth soccer players. J Hum Kinet 62: 1-10.

32. Dalleau G, Belli A, Viale F, Lacour JR, Bourdin M (2004) A simple method for field measurements of leg stiffness in hopping. Int J Sports Med 25(3): 170-176.

33. Flanagan EP, Comyns TM (2008) The use of contact time and the reactive strength index to optimize fast SSC training. Stren Cond J 30(5): 32-38.

34. Lloyd RS, Oliver JL, Hughes MG, Williams CA (2009) Reliability and validity of field-based measures of leg stiffness and reactive strength in youths. J Sports Sci 27(14): 1565-1573.
35. Hůlka K, Lehnert M, Bělka J (2017) Reliability and validity of a basketballspecific fatigue protocol simulating match load. Acta Gymnica 47(2): 92 98.

36. Hopkins WG, Marshall SW, Batterham AM, Hanin J (2009) Progressive statistics for studies in sports medicine and exercise science. Med Sci Sports Exerc 41(1): 3-12.

37. Hunter I, Smith GA (2007) Preferred and optimalstride frequency, stiffness and economy: changes with fatigue during a 1 h high-intensity run. Eur J Appl Physiol 100(6): 653-661.

38. De Ste Croix MBA, Hughes JD, Lloyd RS, Oliver JL, Read PJ (2017) Leg stiffness in female soccer players: inter-session reliability and the fatiguing effects of soccer-specific exercise. J Stren Cond Res 31(11): 3052-3058
Creative Commons Attribution 4.0 International License

For possible submissions Click Here

\section{Submit Article}

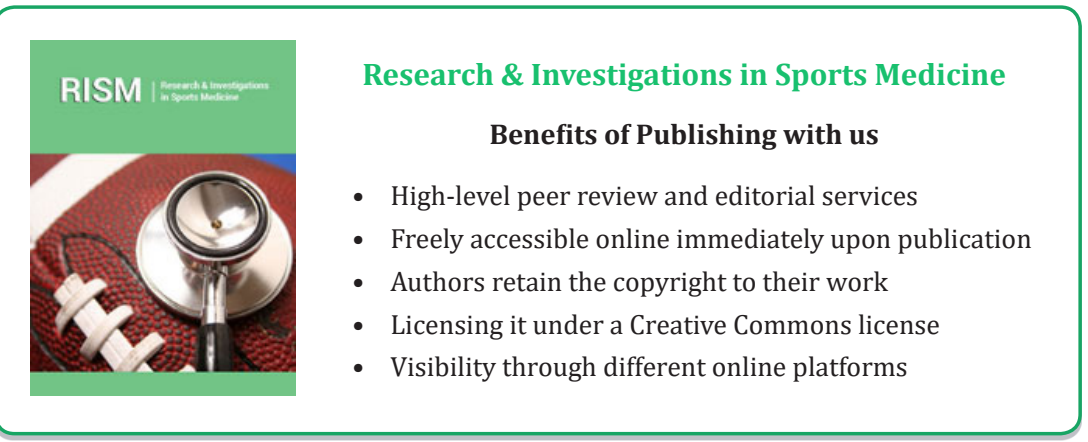

